\title{
Intussusceptive angiogenesis and its role in vascular morphogenesis, patterning, and remodeling
}

\author{
Andrew N. Makanya $\cdot$ Ruslan Hlushchuk • \\ Valentin G. Djonov
}

Received: 18 December 2008/Accepted: 25 December 2008/Published online: 5 February 2009

(C) Springer Science+Business Media B.V. 2009

\begin{abstract}
New blood vessels arise initially as blood islands in the process known as vasculogenesis or as new capillary segments produced through angiogenesis. Angiogenesis itself encompasses two broad processes, namely sprouting (SA) and intussusceptive (IA) angiogenesis. Primordial capillary plexuses expand through both SA and IA, but subsequent growth and remodeling are achieved through IA. The latter process proceeds through transluminal tissue pillar formation and subsequent vascular splitting, and the direction taken by the pillars delineates IA into overt phases, namely: intussusceptive microvascular growth, intussusceptive arborization, and intussusceptive branching remodeling. Intussusceptive microvascular growth circumscribes the process of initiation of pillar formation and their subsequent expansion with the result that the capillary surface area is greatly enhanced. In contrast, intussusceptive arborization entails formation of serried pillars that remodel the disorganized vascular meshwork into the typical tree-like arrangement. Optimization of local vascular branching geometry occurs through intussusceptive branching remodeling so that the vasculature is remodeled to meet the local demand. In addition, IA is important in creation of the local organspecific angioarchitecture. While hemodynamic forces
\end{abstract}

A. N. Makanya · R. Hlushchuk · V. G. Djonov $(\bowtie)$

Department of Medicine, Institute of Anatomy, Fribourg

University, Rte Albert Gockel 1, CH-1700 Fribourg, Switzerland

e-mail: valentin.djonov@unifr.ch

A. N. Makanya

Department of Veterinary Anatomy \& Physiology, University of Nairobi, Riverside Drive, P.O. Box 30197-00100, Nairobi, Kenya have proven direct effects on IA, with increase in blood flow resulting in initiation of pillars, the preponderant mechanisms are unclear. Molecular control of IA has so far not been unequivocally elucidated but interplay among several factors is probably involved. Future investigations are strongly encouraged to focus on interactions among angiogenic growth factors, angiopoetins, and related receptors.

Keywords Intussusceptive angiogenesis .

Vascular growth · Vascular morphogenesis - Sprouting . Vascular remodeling

\section{Definition of intussusceptive angiogenesis}

Intussusceptive angiogenesis defines the process in which transluminal tissue pillars develop within capillaries, small arteries, and veins and subsequently fuse, thus delineating new vascular entities or resulting in vessel remodeling. The concept of intussusceptive angiogenesis was first floated by Caduff et al. [1] when they encountered several tiny holes in the vascular casts of developing pulmonary vessels. They postulated the holes to represent spaces for tissue posts that had been inserted into the vascular lumina and that were digested away during tissue corrosion. These authors coined the name 'intussusceptional angiogenesis' to describe this process of 'in-itself' vascular growth. This terminology was modified to intussusceptive angiogenesis by Burri and Tarek [2] who demonstrated the tissue posts to be pillars in the vascular lumina of developing vessels by serial sectioning. Such pillars were likened to slipping of a piece of tissue into another one and presence of such pillars is, therefore, the quintessence of intussusceptive angiogenesis [3]. 


\section{Mechanisms of intussusceptive angiogenesis}

Pillar formation, the hallmark of intussusceptive angiogenesis, follows precise stages that result in establishment of a tissue post across the vascular lumen. Protrusion of opposite sides of the culprit vascular walls of the same capillary is followed by establishment of interendothelial cell contacts (Fig. 1). Reorganization of the endothelial cell junctions precedes perforation of the bilayer by invading interstitial tissue, pericytes, and myofibroblasts. Collagen fibrils are deposited in the new tissue post that leads to subsequent expansion and ultimate formation of tissue meshes (Fig. 1). Inauguration of pillars on resin intravascular casts is recognized as tiny shallow depressions on the surface of such casts. Larger tissue pillars appear as deep broader holes on the casts and such are differentiated from tissue meshes purely by their sizes, with all holes $<2.5 \mu \mathrm{m}$ in diameter being taken to represent tissue pillars (Fig. 1). Unlike sprouting, intussusceptive angiogenesis has two paramount advantages: firstly, it is achieved at a relatively low rate of endothelial cell proliferation and, secondly, it is accomplished in a relatively short time. In addition, IA is achieved at low vascular permeability with minimal tissue degradation.

In the chick chorioallantoic membrane (CAM), it has been demonstrated that endothelial cell proliferation declines between days 10 and 11 of incubation, when intussusceptive angiogenesis reaches its peak [4]. Further evidence for the non-proliferative nature of IA was adduced in the developing rat lung when the capillary volume and surface area were shown to increase 35- and 20 -fold, respectively [5, 6], with the virtual absence of endothelial cell proliferation [7]. During IA vascular expansion entails thinning and spreading of the existing endothelial cell population [8], a phenomenon that is also demonstrated in the alveolar epithelial cells of the developing marsupial lung [9]. In the CAM, average endothelial cell thickness is reduced by $>50 \%$ during IA [10], plausibly by thinning and spreading of the cells as a result of redistribution of the cytoplasm and cell organelles.

Unambiguous identification of pillars requires specific three-dimensional visualization techniques or even a combination of such techniques. Intravascular casting coupled with serial sectioning for light or transmission electron microscopy and demonstration of pillars or use of confocal laser scanning microscopy avail indubitable evidence for the presence of transluminal pillars [11-13]. A combination of morphological evidence with in vivo observations helps to match time-course events of intussusception with structural alterations (Fig. 1).

Unequivocal evidence for intussusceptive angiogenesis was demonstrated in vivo in developing CAM vessels where pillars were seen to appear as dark spots on blood vessels (Fig. 1). Procurement of the vessel region and subsequent serial sectioning revealed the dark spots to be tissue pillars (Fig. 1) with the archetypical structural characteristics $[12,14]$. Three-dimensional methods, such as magnetic resonance imaging (MRI), micro-computer tomography, angiography, and ultrasonography, lack the $1 \mu \mathrm{m}$ minimum resolution requisite for discerning pillars. Presence of septal tissue between two capillary lumina in single two-dimensional sections does not necessarily represent pillars (however small the septum may be), because such could be just two distinct but closely associated capillaries.

\section{Phases and phenotypes of intussusceptive angiogenesis}

Intussusceptive angiogenesis may be divided into three major phases depending on the outcomes or phenotypes accomplished at the end of the processes [11, 14-17]. Despite these disparate phases, the fundamental denominator to all of them is the formation of tissue pillars, the differences being inherent in the direction and arrangement of such pillars and hence the outcome accomplished. The three phases include intussusceptive microvascular growth (IMG), intussusceptive arborization (IAR), and intussusceptive branching remodeling (IBR). In developing organs, the three phases have been seen to initially occur in tandem, becoming contemporaneous later in development. IMG inaugurates the primordial capillary network expansion while IAR adapts the vasculature into the typical vascular tree pattern [11, 12, 18, 19], IBR finally remodels the vasculature to optimum local perfusion requirements. Normally, IA supplants sprouting angiogenesis after the establishment of the basic plexus but remodeling of the vasculature is achieved through IBR $[9,14,18]$.

Intussusceptive microvascular growth encompasses the process of initiation of pillars and their subsequent expansion with the result that the capillary surface area is greatly increased [12] (Fig. 2). Specific organ angioarchitecture is accomplished through deft remodeling of the blood vessels. In the embryonic avian metanephric kidneys, initial large blood vessels that form the primitive glomerular tufts are split in the middle through the processes of intussusception [19]. These entail arrangement of tissue pillars in line along the longitudinal axis of the vessel, their subsequent fusion, and thus delineation of two branches with result that two daughter glomeruli are formed [19]. In the developing vasculature of the retina, lung, muscle, etc., different pillar arrangement and fusion patterns determine the organ-specific angioarchitecture. Numerous reports in literature indicate that intussusceptive angiogenesis occurs in many developing organs [14], but in some situations it has not been out-rightly recognized and 


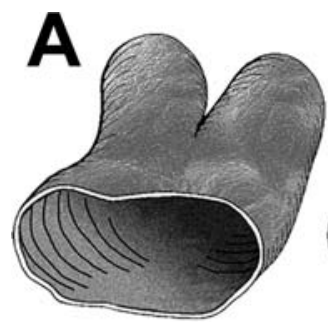

a

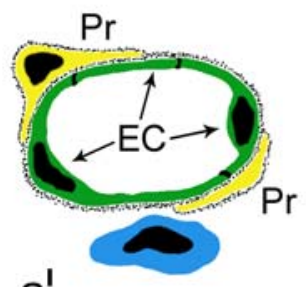

$a^{\prime}$
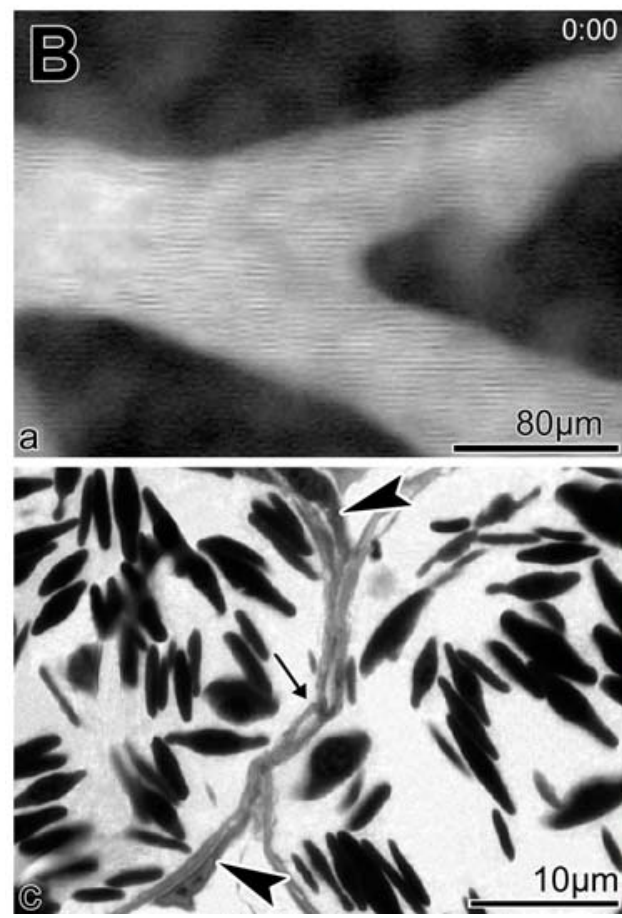

Fig. 1 Demonstration of the mechanisms involved in pillar formation. A $(a-d)$ Three-dimensional schema illustrating the steps in the formation of transluminal pillars during intussusceptive angiogenesis. The process begins with the protrusion of portions of the walls from opposite sides into the vessel lumen $(a, b)$. After contact has been established and "corroborated" $(c)$, the endothelial bilayer becomes perforated centrally and a transluminal pillar is formed $(d) .\left(a^{\prime}-d^{\prime}\right)$ Two-dimensional representation of the events depicted in $(a-d)$ above. Endothelial cells $(E C)$ situated on opposite sides of a capillary protrude into its lumen until they contact each other $\left(a^{\prime}-c^{\prime}\right)$. Once established, this contact is fortified by the formation of interendothelial junctions and then reorganized in such a manner that the endothelial bilayer is perforated centrally. The endothelial cells then retract, and the newly formed pillar increases in girth after being invaded by fibroblasts $(F b)$ and pericytes $(P r)$, which lay down

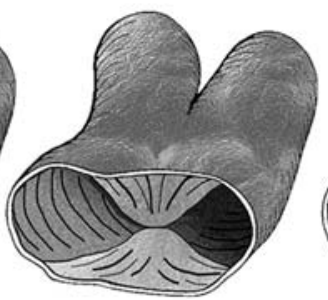

C

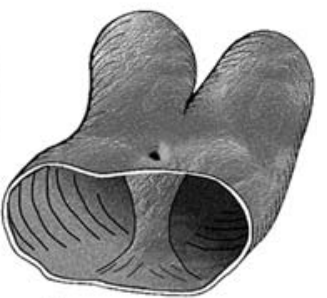

d
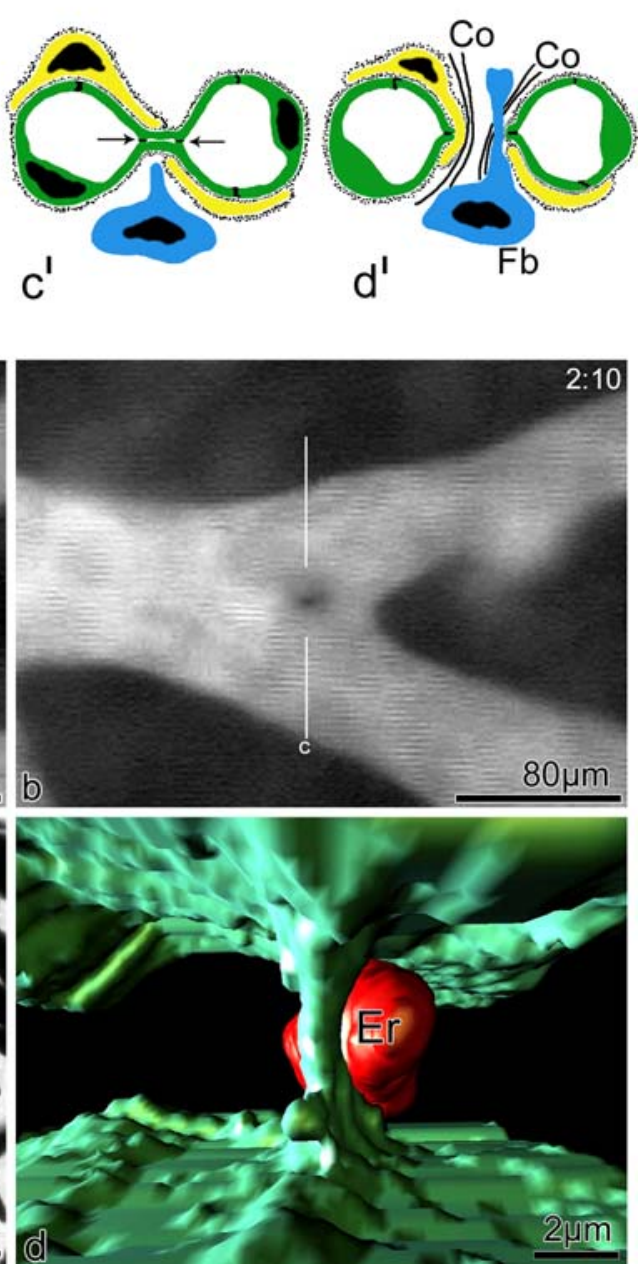

collagen fibrils ( $C o$ in $d^{\prime}$ ); modified from Djonov et al. [8]. B ( $a$ and $b$ ) In vivo video images illustrating pillar formation at a venous bifurcation in the chick embryo CAM. After half an hour of surveillance, a dark spot became visible and continued to increase in size. The region with the dark spot was procured after $2 \mathrm{~h}$ and serial semithin sections made to demonstrate the pillar. (c) A semithin section through the dark spot revealed the presence of an hour-glassshaped pillar, created by the simultaneous protrusion of endothelial cells from opposite sides of the vascular wall into the lumen. The intensely stained zone (arrow) represents an intercellular junction within the endothelial bilayer. Notice some pericytes (arrowheads) invading the pillar. (d) Three-dimensional reconstruction of a transcapillary pillar based on ultrathin (TEM) serial sections obtained from chick CAM. The pillar is indicated with an arrow while Er denotes an erythrocyte. Obtained from [12], with permission 


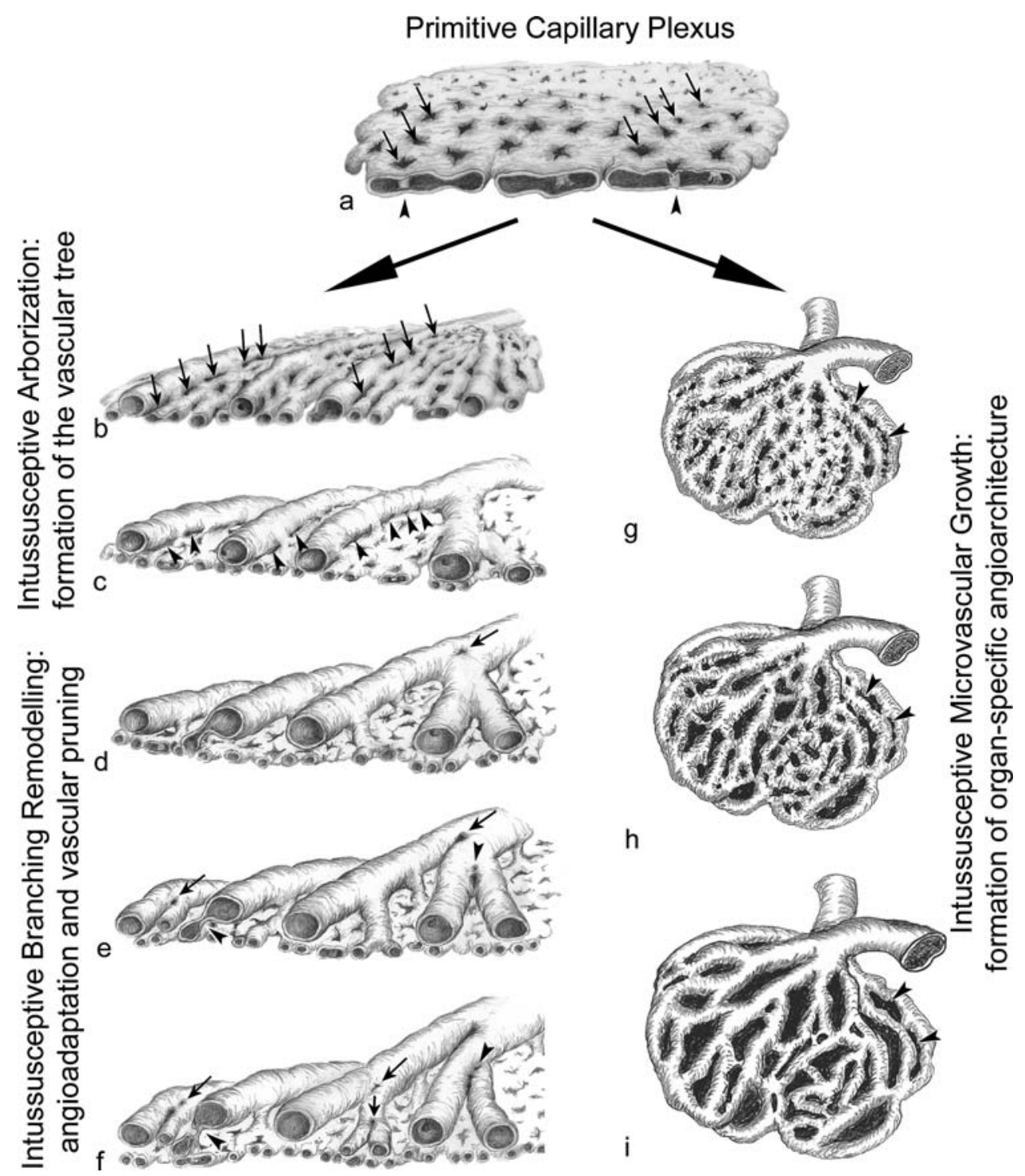

Fig. 2 A schematic drawing showing the phases and phenotypes of intussusceptive angiogenesis (not drawn to scale). Modified from [8]. a The initial capillary plexus is a disorganized meshwork without a definite phenotype. The development of this meshwork proceeds though insertion of new pillars (arrows), which result in rapid expansion of the capillary plexus. Arrowheads indicate intraluminal appearance of the pillars. b, c From the disorganized capillary meshwork, IAR segregates the various vessel generations by formation of 'vertical' pillars in rows (arrows in b) and narrow tissue septa formed by pillar reshaping and pillar fusions segregate the new vascular entities. Subsequently, formation of 'horizontal pillars' and folds (arrowheads in c), which separates the new vessels from the capillary plexus. $\mathbf{d}-\mathbf{f}$ The vasculature is finally adapted by IBR to suit the local perfusion demands. This entails modification of the branching angles and the diameters of the vessels by insertion of

has been given different names such as longitudinal splitting [20], longitudinal division [21], intraluminal splitting angiogenesis [22], or internal division [23]. Notably, the direction taken by pillars in relation to the axis of the remodeling vessels depends on the targeted outcomes. transluminal pillars at branching points (arrows). Expansion and fusion of such pillars relocate the branching angle proximally with a concomitant change in blood flow properties. Part of the vascular remodeling by IBR involves severance of putative superfluous vessels, a process known as vascular pruning, which entails formation of eccentric pillars across the target branch (arrowheads in $\mathbf{e}, \mathbf{f}$ ), their subsequent augmentation and fusion resulting in ablation of the vessel. $\mathbf{g}$-i Demonstration of intussusceptive microvascular growth (IMG) in the kidney glomerulus. Regardless of the organ, IMG proceeds through pillar initiation (arrowheads in $\mathbf{g}$ ), pillar expansion (arrowheads in h), and ultimate fusion (arrowheads in i). In this way, the primordial simple capillary network is greatly expanded with delineation of new vascular segments and, depending on the pillar fusion pattern, the organ-specific angioarchitecture is formed

Pillars arranged in rows along the vessel length, eventually result in longitudinal vessel division. Despite some reports to the contrary [21], there is no evidence that longitudinal vessel division is any different from intussusceptive angiogenesis. Indeed, Prior et al. [24] indicate that all these are 

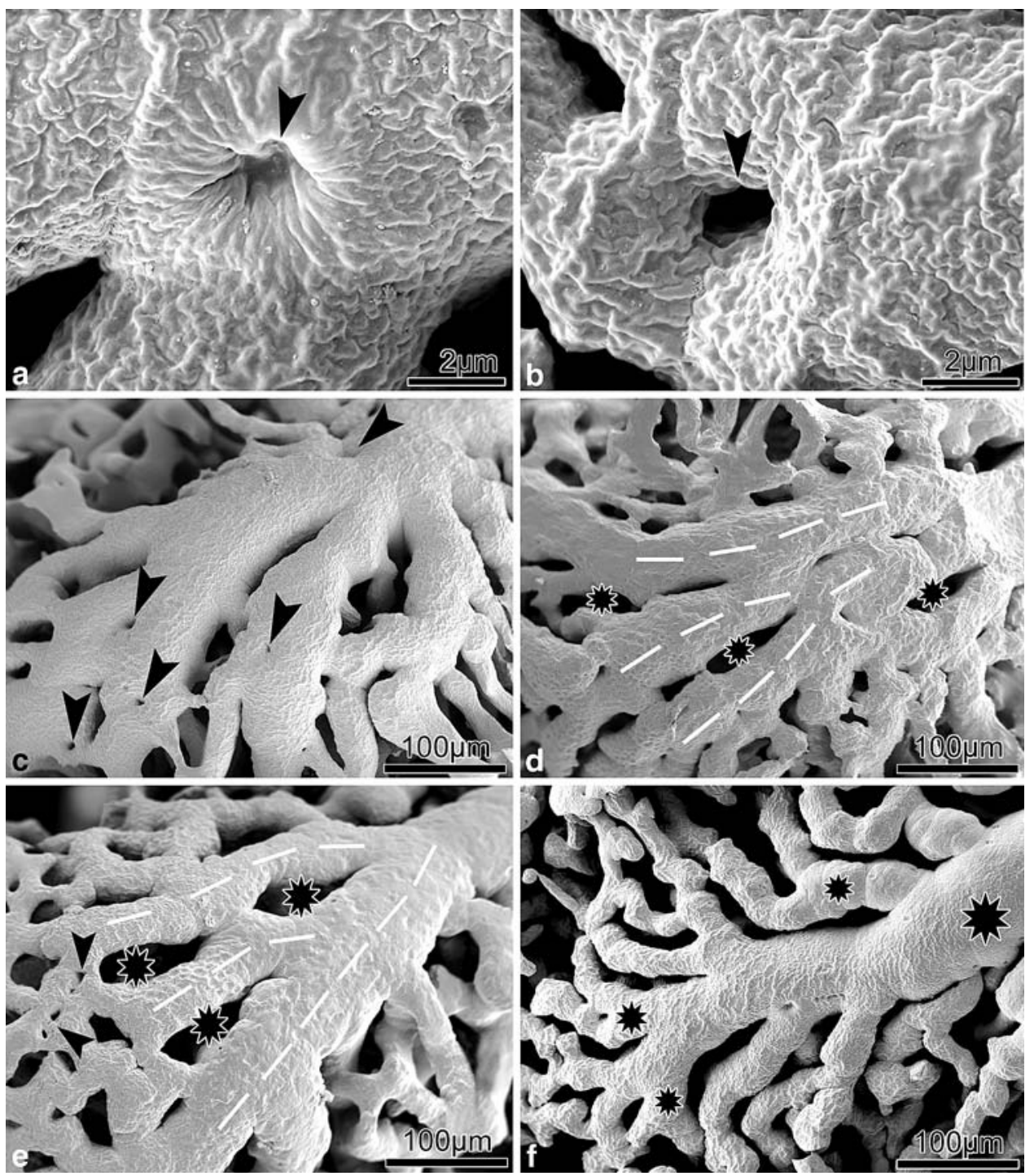

Fig. 3 a-f Vascular corrosion casts illustrating the process of intussusceptive microvascular growth and intussusceptive arborization in the E15 metanephric avian kidney. a Incipient pillars appear as small depressions on the surface of the blood vessel cast (arrowhead). Such a depression indicates the initial stages of pillar formation (see also Fig. 1A). b As the two opposite components of the pillar approximate, there is fusion and subsequent perforation so that the pillar is now represented by a hole that pierces through the vessel cast (arrowhead). c-f Low magnification microvascular casts showing the various stages in IMG. Incipient pillars are represented by depressions

components of the intussusceptive angiogenesis process. In the capillaries of skeletal muscle, pillars appear more elongated than in other tissues due to the parallel arrangement of myofibres so that the spaces left for the capillaries have a general longitudinal disposition [3].

Intussusceptive arborization defines the process which delineates smaller generations of future feeding and draining vessels and contributes immensely to the formation and expansion of the vascular tree. The original pattern

or small holes in the cast (arrowheads in c). Notice the irregular nature of the resultant vessels. Subsequently, pillars increase in girth and fuse (asterisks in $\mathbf{d}$ and $\mathbf{e}$ ) and in so doing delineate new vascular entities (interrupted lines in $\mathbf{d}$ and $\mathbf{e}$ ). Further expansion of pillars (asterisks in $\mathbf{e}$ ) separates out the newly formed vessels (asterisks in $\mathbf{f}$ ). Note that new pillars (arrowheads in e) now tend to form lower down in the vascular tree as the network matures. Microvascular maturation (f) results in the typical vascular tree hierarchy and vessel branches (asterisks) are virtually devoid of pillar holes; after Djonov and Makanya [3]

of blood vessels formed either through vasculogenesis or through sprouting angiogenesis is a disorganized meshwork and hardly resembles the tree-like arrangement of the mature vasculature $[14,18]$. The adaptation of the organ vascular tree is achieved through IAR, which entails formation of serried "vertical" pillars that demarcate lower generations of vessels (Figs. 2, 3). Numerous circular pillars are formed in rows, thus demarcating future vessels; pillar reshaping and pillar fusions result in formation of 
Fig. 4 a-f Vascular casts illustrating the process of intussusceptive branching remodeling in the chick CAM $(\mathbf{a}-\mathbf{c})$ and metanephric kidney (d-f). a-c Vessel branching angle modification by IBR. The process inaugurates with formation of a small pillar (arrowhead in a), which expands until the connection of the vessels distal to the point of pillar initiation is severed $(\mathbf{b}, \mathbf{c})$ with the result that the bifurcation point is shifted proximally and the branching angle is reduced. $\mathbf{d}-\mathbf{f}$ Vascular casts of the chick glomerular capillaries at E20 showing series of pillars (arrowheads in e and f) and longitudinal folds of the endothelial wall (arrows) at vascular bifurcations, indicating ongoing intussusceptive branching remodeling; after Djonov and Makanya [3]

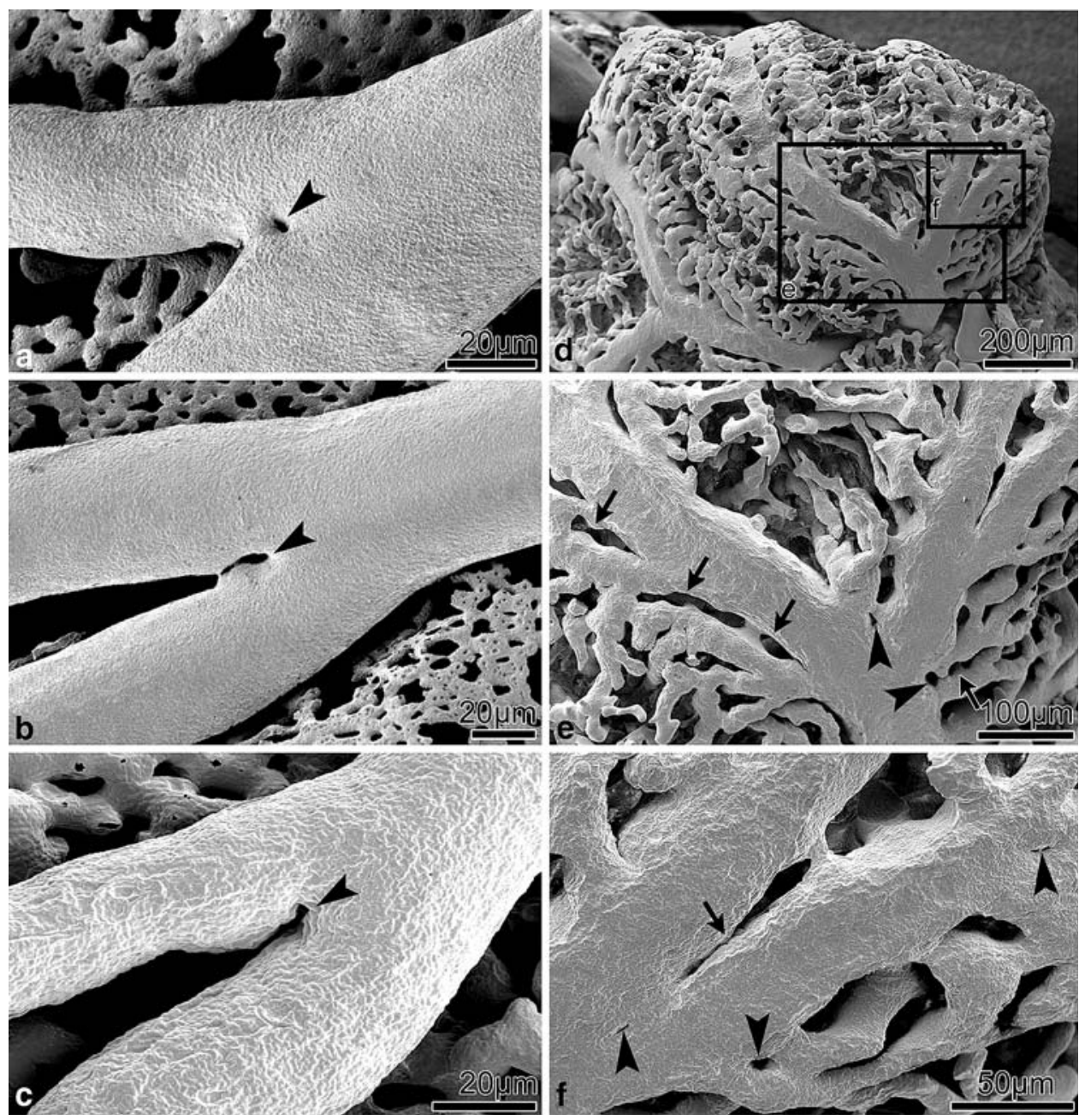

narrow tissue septa. There is delineation, segregation, growth, and extraction of the new vascular entities by merging of septa [12]. New branching generations are formed by successive recapitulation of the process, complemented by growth and maturation of all components. Any remnant bridges interconnecting new vascular entities are severed by formation of horizontal pillar folds, thus the feeding and draining vessels are separated from the capillary plexus so that the two vessel categories are in different planes (Fig. 2). In the chicken lung, this process results in a unique vessel arrangement at the parabronchial level whereby the arterial system remains external to the gas exchange mantle, thus allowing space for interaction of the gas-exchanging units (Makanya and Djonov, unpublished data).

Intussusceptive branching remodeling describes the processes that result in adaptation of the architecture and number of vascular branches to optimum local requirements [14]. Accomplishment of IBR occurs via transluminal pillars that are formed close to arterial or venous bifurcation sites (Figs. 2, 4). Enlargement of such pillars, their subsequent approximation and fusion, and also fusion with the connective tissue at the bifurcation narrows the bifurcation angle by relocating the branching point proximally [14]. Symorphosis is a concept that postulates a match between function and structural design [25], while Murray's law envisages an ideal situation of minimum power consumption and constant shear stress in blood flow [26]. In vascular remodeling, IBR may lower blood pressure by decreasing the branching angle. This is achieved by relocation of the branching point proximally. Relocation of bifurcation angles has been reported in the cremaster muscle of the golden hamster following alterations of blood flow [27] and in retinal vessels of hypertensive human subjects [28].

In remodeling mature vessels, IBR severs superfluous vessels, a process known as intussusceptive vascular pruning (IPR). The latter process is achieved through eccentric formation of pillars in rows across the breadth of the target vessel at bifurcation sites (Figs. 2, 5). Expansion and subsequent fusion of pillars results in reduced blood flow, the consequences being regression, retraction, and 
Fig. 5 A schematic diagram presented by Clarke and Clarke [32] showing vascular remodeling in the rabbit ear. Some of their excellent drawings reveal small eccentric ellipsoid pillars (denoted here by arrowheads) that split the vessel lumen at the branching points. Fusion of these pillars leads to a separation of the lateral branch within 2 days and subsequent intussusceptive pruning and atrophy of the affected vessel branch. This illustration is probably the first documented evidence of branching remodeling, although it was not explicitly recognized as such at the time. Reproduced from Clarke and Clarke [32]. Figure not drawn to scale
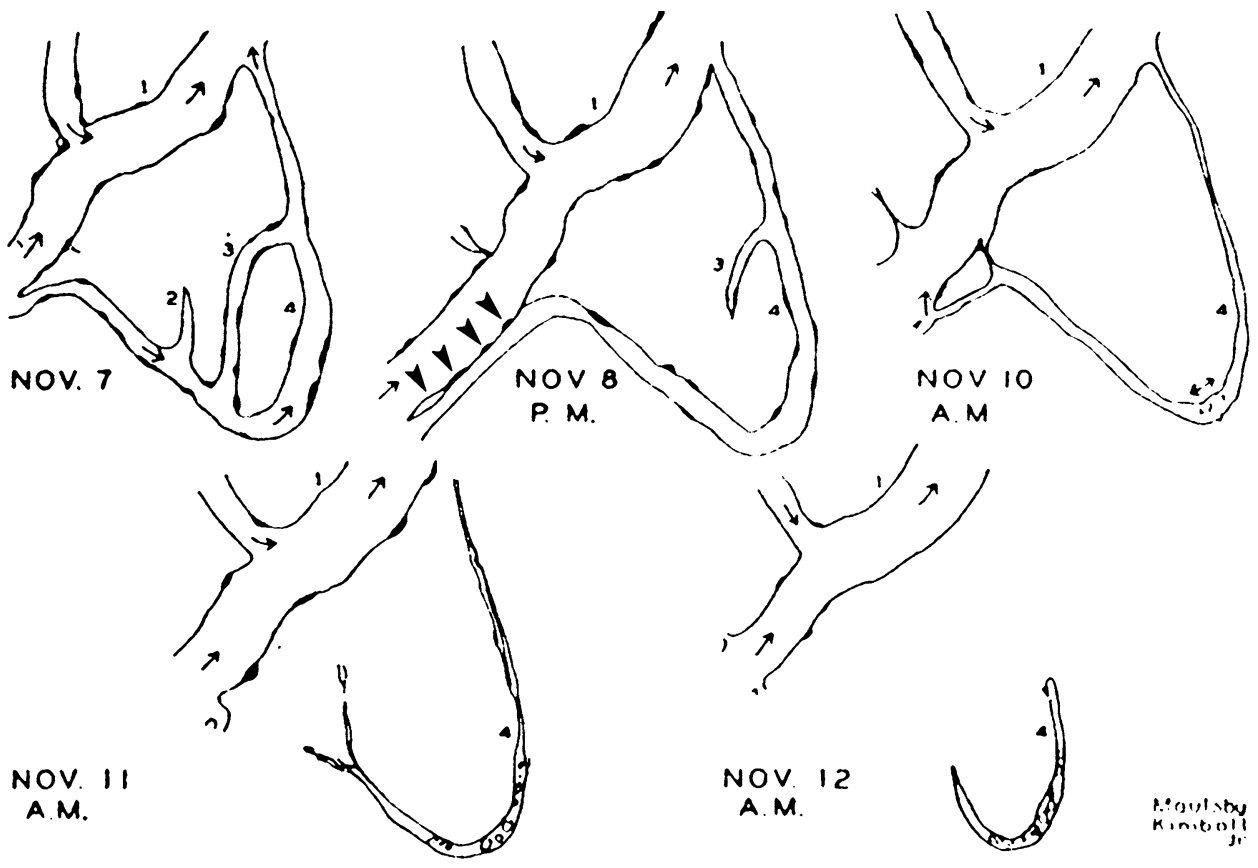

atrophy of the affected vessel (see review, [3]). The phenomenon of vascular pruning was first reported in retinal vessels long before IA was described [29], and a classical description of the process and mechanisms involved was provided by Djonov et al. [14] and has been elucidated in several reviews [3, 8, 16, 17]. It has also been demonstrated in the remodeling vasculature of the developing metanephros [19] and the maturing CAM vasculature [14]. IPR is related to reduction in blood flow and oxygen tension. In the absence of blood flow, pruning was seen to be accelerated [30] while hyperoxia resulted in down-regulation of VEGF and subsequent vascular pruning [31]. Vessel pruning was demonstrated many decades ago by Clarke and Clarke [32] when they documented that the vasculature of an inflamed rabbit ear reverted to a simple architecture after relief from inflammation (Fig. 5).

Another type of pruning achieved through leukocytes has been demonstrated in the retina [33]. Leukocytes adhere to the vessels and this leads to Fas-ligand-mediated endothelial cell apoptosis. Whether this has any relation to pillar formation is not clear. Generally, reduction of vascular branches has been referred to as vascular pruning, but it is only on few occasions that IPR per se has been demonstrated [14, 31].

\section{Temporospatial distribution of intussusceptive and sprouting angiogenesis}

It is noteworthy that IA occurs only on pre-existing vasculature, formed either through sprouting angiogenesis or through vasculogenesis. As has been shown in several studies, IA is preceded by SA and forms an important part of the vascular remodeling. In the much studied CAM, blood vessels grow by SA in its first phase of development (E5-E7) and in the second phase (E8-E12) they grow mainly by IMG while the final phase grows without a substantial increase in vascular complexity [4]. It was further shown that expansion of the CAM vasculature during the second phase was via IAR while IBR remodeled the vessels during the maturation phase [14, 17].

In the growing rat, the mammary vasculature during the pubertal, adult virgin, and early pregnancy stages grows by sprouting angiogenesis, after which this process gives way to massive intussusceptive angiogenesis [13]. In the ephemeral ovarian follicles, sprouting angiogenesis characterizes the initial phase of development and this is soon supplanted by IA, which expands and remodels the vasculature towards the time of follicular maturation [34]. Similarly, in the evanescent avian mesonephros, SA precedes and is supervened by IA. However, IA is short-lived, starting at E7, soon after the primitive mesonephric plexus is established and continuing to E11, whence obvious signs of vascular degeneration preponderate [19]. In the chick embryo mesonephros, vascular development has a craniocaudal orientation; sprouting establishes a denser network cranially by stages 29 (E6) and 30 (E7). IA gradually starts to replace SA so that the cranial aspect of the mesonephric vasculature casts has many pillar holes while the caudal part has many sprouts [19]. This shows that the two processes can be contemporaneous in the same organ, albeit spatially discrete. The subsequent phases of vascular 
remodeling fail to inaugurate in the mesonephros since it starts to degenerate [19].

In the non-evanescent avian metanephros, SA is the preponderant mode of vessel development up to E13 when this is supervened by IA [19]. Subsequently, the vasculature is expanded through IMG and IAR accomplishes the vascular tree pattern [19]. Characteristic steps of IBR delineate individual glomeruli from large supplying vessels. This is accomplished through formation of pillars in rows along the longitudinal median axis of large vessels destined to form glomeruli. Fusion of such pillars results in two vessels each of which proceeds to form a new glomerulus [19].

The effect of IA does not become apparent in the embryonic avian lung until about the 15th day of incubation, when there is an angiogenic switch with an upsurge in the number of transluminal pillars [18]. Initially, the vessels develop by sprouting angiogenesis, guided by the air conduits that develop ahead of the vasculature but as soon as the basic network is established, IA starts to delineate the fine supplying and draining vessels at the arterial and venous sides, respectively [18]. In addition, IA is responsible for remodeling the fine capillaries that interlace with air capillaries to form the thin blood-gas barrier characteristic of the avian lung [18].

In pathological conditions that are characterized by excessive angiogenesis such as psoriasis, rheumatic disease, retinopathy and tumorigenesis, IA is suspected as one of the participating processes but this far overwhelming evidence has only been adduced for tumorigenesis [35, 36]. In a recent study, it has been shown that radiotherapy of tumors or treatment with an inhibitor of VEGF tyrosine kinase results in transient reduction in tumor growth rate with decreased tumor vascularization followed by posttherapy relapse with extensive IA [35]. The switch from SA to IA is a part of the angioadaptive mechanism responsible for the tumor recovery in the early phase after anti-angiogenic treatment or radiotherapy [35].

\section{Control of intussusceptive angiogenesis}

Intussusceptive angiogenesis occurs during embryonic development, in physiological adaptations as may be seen in exercised muscles and also in pathological situations such as tumorigenesis (for details see reviews [3, 8, 15]). The stimuli for this type of angiogenesis are either physiological, such as alterations in blood flow, as occurs during exercise, or may be local biochemical alterations, as may be envisaged in increased expression of genes that transcribe molecules specific for endothelial cell growth. No specific roles of any such molecules, however, have been identified but a few have been seen to be up-regulated during IA.

\section{Hemodynamic control}

Blood flow within vessels results in stress, referred to as shear stress. Shear stress may be laminar, thus acting tangentially or parallel to the endothelial surface, or otherwise is oscillatory, also regarded as turbulent (see review by Davies [37]). Shear stress, defined as the force per unit area of the endothelial luminal surface imparted by flowing blood in capillaries, acts tangentially on the vascular wall. Enhanced laminar shear has been shown to preserve integrity of the walls of vessels, in part through Ets-1 dependent induction of protease inhibitors [38]. In addition, laminar shear stress is known to inhibit tubule formation and migration of endothelial cells by an angiopoietin-2 (Ang-2)-dependent mechanism, which entails down-regulation of Ang-2 [39] and is associated with IA. Laminar shear stress also increases endothelial actin filament bundles [40], but whether these fibers are important in the endothelial cell movements during pillar formation is unclear. In contrast, turbulent shear stress [41, 42] results in angiogenesis and remodeling of the vessels with an increase in cell proliferation and migration, a process characteristic of sprouting angiogenesis. Oscillatory shear stress results in the production of Ang-2 in endothelial cells and plays a critical role in migration and tubule formation and may be a culprit in diseases with disturbed flow and angiogenesis [39].

The role of hemodynamics in control of IA was demonstrated by clamping of one of the dichotomous branches of an artery in the developing CAM microvasculature [14]. Increase in blood flow and pressure in the cognate artery resulted in an almost immediate effect on branching morphology with pillars beginning to appear in 15-30 min of clamping and a concomitant reduction in branching angles by about $20 \%$ after $40 \mathrm{~min}$ [14]. This indicates that alterations in hemodynamics result in an immediate vascular adaptation. Egginton et al. [43] reported that capillary growth in muscles with increased blood flow occurred through intraluminal splitting, with no sprouting, a mechanism typical of IA. Notably, in the latter case, there was absence of endothelial cell proliferation or breakdown of the basement membrane. Indeed, increase in flow velocity increases shear stress.

Mechanical stretch of vessels may result in expansion, as may occur during increased flow probably providing the substrate for intussusceptive microvascular augmentation. Indeed, capillary network remodeling occurs in response to the mechanical forces of increased shear stress and cell stretch [24, 44]. Stretch behaves much more like oscillatory shear stress and results in up-regulation of MMPs and VEGF with consequent sprouting angiogenesis [45].

Changes in shear stress are sensed by the endothelial cells and the signal is transduced by molecules such as 


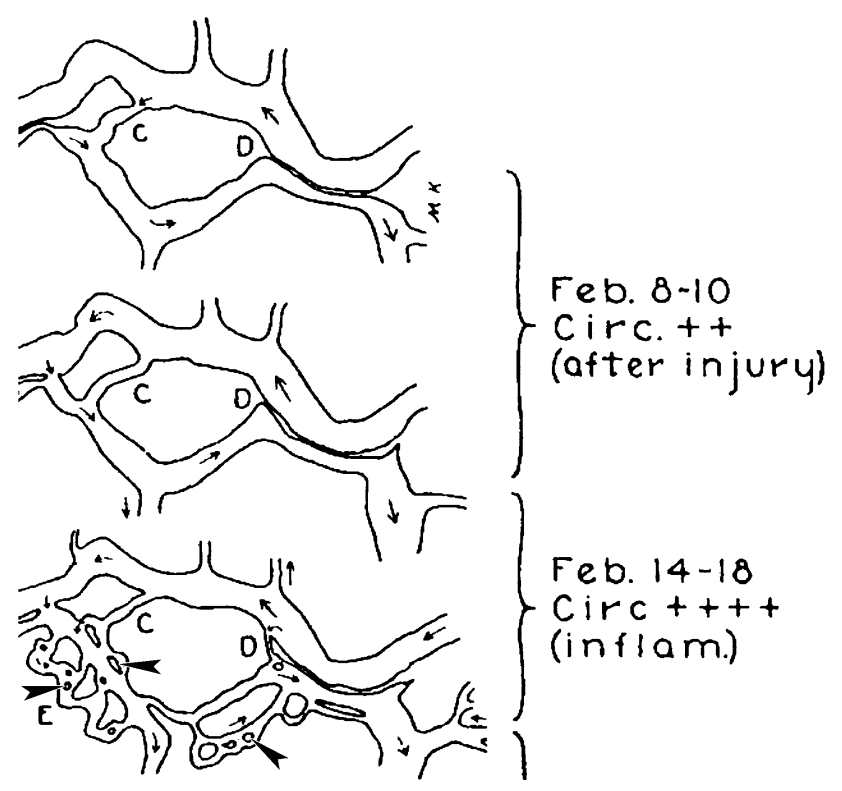

Fig. 6 A schematic diagram showing vascular remodeling in the rabbit ear following injury and subsequent inflammation. Within 4 days, increased blood flow resulted in formation of many tiny vascular loops reminiscent of small and larger pillars with an increased complexity of the vascular network. The spaces on the vascular loops (arrowheads) are indicative of intussusceptive growth, although they were not recognized as such then (not drawn to scale). The arrows indicate direction of blood flow and the plus signs (+) indicate blood velocity. Modified from Clarke and Clarke [52]

PECAM/CD31 [46]. This proceeds through a cascade of events that result in transcription of many molecules involved in angiogenesis such as eNos and growth factors [47, 48]. Shear stress is known in vitro to activate the vascular endothelial growth factor receptor-2 (VEGFR-2) pathway [49] and can, indirectly, influence the mitogenic effect of VEGF via nitric oxide [50]. During development, sensitivity to the local hemodynamic environment facilitates assembly and remodeling of appropriate microvascular network structures and may continue to be critical in maintaining and remodeling capillary networks in adulthood [51]. The essential role played by hemodynamics was demonstrated many decades ago by Clarke and Clarke [52] when they illustrated increase in vessel complexity as a result of enhanced blood flow subsequent to inflammation. The latter authors showed that such an alteration in hemodynamics resulted in formation of secondary loops and that the vasculature reverted to the simple architecture after relief from inflammation (Fig. 6). The same authors had earlier demonstrated that a vessel branch became separated completely from the main vessel within 3 days, and subsequently became obliterated [32] but they never recognized the role played by eccentrically located pillars illustrated in their schema as small loops (Fig. 6). The role of such pillars in vascular pruning has since been unequivocally elucidated $[3,8,14]$.

\section{Molecular control}

Investigations into molecular control of IA need to take into consideration the mechanisms that entail pillar formation, the archetype of this process. The primeval indicators of pillar initiation are intraluminal endothelial protrusions, discerned as minute shallow depressions on intravascular casts or projections into the vessel lumen in serial sections. Such protrusions are followed by endothelial cell contacts, reorganization of endothelial cell junctions and invasions of the pillar core by myofibroblasts and pericytes, which lay down collagen fibrils $[2,3]$. The most important growth factor in angiogenesis is VEGF and is known to support both sprouting and intussusceptive angiogenesis [18, 19, 22]. Other endothelial growth factors implicated in IA include bFGF $[18,19]$ and PDGF-B [18].

It has been shown that bFGF up-regulates PDGFR- $\alpha$ and $-\beta$ expression levels in the newly formed blood vessels and PDGF-AB and $-\mathrm{BB}$ act through PDGFR- $\beta$ to enhance vessel stability [53]. The factors that initiate transluminal pillars are unknown. However, PDGF and Ang-2 are both known to be important in pericyte recruitment [54-56] and may therefore play a role in pillar formation. In knock-out mice lacking angiopoetin-1 (Ang-1) and Tie-2, vessel growth is arrested at an early stage of development and further remodeling does not occur [57]. On injection of a monoclonal antibody against PDGFR- $\beta$, the receptor for PDGF-B, in murine neonates completely blocks mural cell recruitment in the developing retinal vessels [58]. PDGF-B promotes pericyte recruitment by stimulating both proliferation and migration of such cells [59]. Angiopoietin-1 cannot initiate angiogenesis; it is constitutively expressed throughout the body and promotes vascular remodeling, maturation, and stabilization of vessels via its Tie-2 receptor [24]. Over-expression of VEGF simultaneously with Ang-1 or Ang-2 results in large vessels and small holes in the casts of capillaries [60], a sign of intussusceptive angiogenesis. Molecules specifically associated with cell migration such as neuropilin, restin, and midkine are down-regulated during the phase of intussusceptive angiogenesis [22]. To date, no direct evidence linking a specific molecule to intussusceptive angiogenesis has been adduced. However, a synergism between VEGF and Ang-1 is highly suspect. Transgenic mice over-expressing VEGF in the skin have numerous tortuous and leaky capillaries whereas those with Ang-1 over-expression have enlarged but less leaky vessels [60-62]. VEGF is essential for early blood vessel formation and mice deficient in VEGF gene die early during embryogenesis with severe defects in blood vessel formation [63, 64]. In contrast, Ang-1 is necessary for later stages of vessel development, and mice with deficiency die of problems associated with vessel 
remodeling and maturation [57]. Future studies on molecular control of intussusceptive angiogenesis are persuaded to focus on the interactions between angiogenic growth factors (especially VEGF), angiopoetins and their cognate receptors.

Acknowledgments The figures in this article were reproduced and/ or modified with the kind permission from the relevant copyright holders. These studies were supported by a Swiss National Science foundation grant to VD.

\section{References}

1. Caduff JH, Fischer LC, Burri PH (1986) Scanning electron microscope study of the developing microvasculature in the postnatal rat lung. Anat Rec 216:154-164. doi:10.1002/ar.1092 160207

2. Burri PH, Tarek MR (1990) A novel mechanism of capillary growth in the rat pulmonary microcirculation. Anat Rec 228:3545. doi:10.1002/ar.1092280107

3. Djonov V, Makanya AN (2005) New insights into intussusceptive angiogenesis. EXS 94:17-33

4. Schlatter P, Konig MF, Karlsson LM, Burri PH (1997) Quantitative study of intussusceptive capillary growth in the chorioallantoic membrane (CAM) of the chicken embryo. Microvasc Res 54:65-73. doi:10.1006/mvre.1997.2022

5. Burri PH, Dbaly J, Weibel ER (1974) The postnatal growth of the rat lung. I. Morphometry. Anat Rec 178:711-730. doi:10.1002/ar. 1091780405

6. Zeltner TB, Caduff JH, Gehr P, Pfenninger J, Burri PH (1987) The postnatal development and growth of the human lung. I. Morphometry. Respir Physiol 67:247-267. doi:10.1016/00345687(87)90057-0

7. Kauffman SL, Burri PH, Weibel ER (1974) The postnatal growth of the rat lung. II. Autoradiography. Anat Rec 180:63-76. doi: 10.1002/ar.1091800108

8. Djonov V, Baum O, Burri PH (2003) Vascular remodeling by intussusceptive angiogenesis. Cell Tissue Res 314:107-117. doi: 10.1007/s00441-003-0784-3

9. Makanya AN, Sparrow MP, Warui CN, Mwangi DK, Burri PH (2001) Morphological analysis of the postnatally developing marsupial lung: the quokka wallaby. Anat Rec 262:253-265. doi:10.1002/1097-0185(20010301)262:3<253::AID-AR1025>3.0. $\mathrm{CO} ; 2-\mathrm{B}$

10. Rizzo V, DeFouw DO (1993) Macromolecular selectivity of chick chorioallantoic membrane microvessels during normal angiogenesis and endothelial differentiation. Tissue Cell 25:847856. doi:10.1016/0040-8166(93)90033-H

11. Djonov VG, Galli AB, Burri PH (2000) Intussusceptive arborization contributes to vascular tree formation in the chick chorioallantoic membrane. Anat Embryol (Berl) 202:347-357. doi: $10.1007 / \mathrm{s} 004290000126$

12. Djonov V, Schmid M, Tschanz SA, Burri PH (2000) Intussusceptive angiogenesis: its role in embryonic vascular network formation. Circ Res 86:286-292

13. Djonov V, Andres AC, Ziemiecki A (2001) Vascular remodelling during the normal and malignant life cycle of the mammary gland. Microsc Res Tech 52:182-189. doi:10.1002/1097-0029 (20010115)52:2<182::AID-JEMT1004>3.0.CO;2-M

14. Djonov VG, Kurz H, Burri PH (2002) Optimality in the developing vascular system: branching remodeling by means of intussusception as an efficient adaptation mechanism. Dev Dyn 224:391-402. doi:10.1002/dvdy.10119
15. Burri PH, Djonov V (2002) Intussusceptive angiogenesis-the alternative to capillary sprouting. Mol Aspects Med 23:S1-S27. doi:10.1016/S0098-2997(02)00096-1

16. Kurz H, Burri PH, Djonov VG (2003) Angiogenesis and vascular remodeling by intussusception: from form to function. News Physiol Sci 18:65-70

17. Burri PH, Hlushchuk R, Djonov V (2004) Intussusceptive angiogenesis: its emergence, its characteristics, and its significance. Dev Dyn 231:474-488. doi:10.1002/dvdy.20184

18. Makanya AN, Hlushchuk R, Baum O, Velinov N, Ochs M, Djonov V (2007) Microvascular endowment in the developing chicken embryo lung. Am J Physiol Lung Cell Mol Physiol 292:L1136-L1146. doi:10.1152/ajplung.00371.2006

19. Makanya AN, Stauffer D, Ribatti D, Burri PH, Djonov V (2005) Microvascular growth, development, and remodeling in the embryonic avian kidney: the interplay between sprouting and intussusceptive angiogenic mechanisms. Microsc Res Tech 66:275-288. doi:10.1002/jemt.20169

20. van Groningen JP, Wenink AC, Testers LH (1991) Myocardial capillaries: increase in number by splitting of existing vessels. Anat Embryol (Berl) 184:65-70. doi:10.1007/BF01744262

21. Egginton S (2008) Invited review: activity-induced angiogenesis. Pflugers Arch. doi:10.1007/s00424-008-0563-9

22. Williams JL, Weichert A, Zakrzewicz A, Da Silva-Azevedo L, Pries AR, Baum O et al (2006) Differential gene and protein expression in abluminal sprouting and intraluminal splitting forms of angiogenesis. Clin Sci (Lond) 110:587-595. doi:10.1042/CS20050185

23. Zhou A, Egginton S, Hudlicka O, Brown MD (1998) Internal division of capillaries in rat skeletal muscle in response to chronic vasodilator treatment with alpha1-antagonist prazosin. Cell Tissue Res 293:293-303. doi:10.1007/s004410051121

24. Prior BM, Yang HT, Terjung RL (2004) What makes vessels grow with exercise training? J Appl Physiol 97:1119-1128. doi: 10.1152/japplphysiol.00035.2004

25. Weibel ER, Taylor CR, Hoppeler H (1991) The concept of symmorphosis: a testable hypothesis of structure-function relationship. Proc Natl Acad Sci USA 88:10357-10361. doi:10.1073/ pnas.88.22.10357

26. Murray CD (1926) The physiological principle of minimum work: I. The vascular system and the cost of blood volume. Proc Natl Acad Sci USA 12:207-214. doi:10.1073/pnas.12.3.207

27. Frame MD, Sarelius IH (1993) Arteriolar bifurcation angles vary with position and when flow is changed. Microvasc Res 46:190205. doi:10.1006/mvre.1993.1046

28. Stanton AV, Wasan B, Cerutti A, Ford S, Marsh R, Sever PP et al (1995) Vascular network changes in the retina with age and hypertension. J Hypertens 13:1724-1728. doi:10.1097/00004872199501000-00008

29. Ashton N (1966) Oxygen and the growth and development of retinal vessels. In vivo and in vitro studies. The XX Francis I. Proctor lecture. Am J Ophthalmol 62:412-435

30. Bongrazio M, Da Silva-Azevedo L, Bergmann EC, Baum O, Hinz B, Pries AR et al (2006) Shear stress modulates the expression of thrombospondin-1 and CD36 in endothelial cells in vitro and during shear stress-induced angiogenesis in vivo. Int $\mathbf{J}$ Immunopathol Pharmacol 19:35-48

31. Dor Y, Porat R, Keshet E (2001) Vascular endothelial growth factor and vascular adjustments to perturbations in oxygen homeostasis. Am J Physiol Cell Physiol 280:C1367-C1374

32. Clarke ER, Clarke EL (1939) Microscopic observations of the growth of blood capillaries in the living mammal. Am J Anat 64:251-299. doi:10.1002/aja.1000640203

33. Ishida S, Yamashiro K, Usui T, Kaji Y, Ogura Y, Hida T et al (2003) Leukocytes mediate retinal vascular remodeling during development and vaso-obliteration in disease. Nat Med 9:781788. doi: $10.1038 / \mathrm{nm} 877$ 
34. Macchiarelli G, Jiang JY, Nottola SA, Sato E (2006) Morphological patterns of angiogenesis in ovarian follicle capillary networks. A scanning electron microscopy study of corrosion cast. Microsc Res Tech 69:459-468. doi:10.1002/jemt.20305

35. Hlushchuk R, Riesterer O, Baum O, Wood J, Gruber G, Pruschy $M$ et al (2008) Tumor recovery by angiogenic switch from sprouting to intussusceptive angiogenesis after treatment with PTK787/ZK222584 or ionizing radiation. Am J Pathol 173:11731185. doi:10.2353/ajpath.2008.071131

36. Patan S, Munn LL, Jain RK (1996) Intussusceptive microvascular growth in a human colon adenocarcinoma xenograft: a novel mechanism of tumor angiogenesis. Microvasc Res 51:260-272. doi:10.1006/mvre.1996.0025

37. Davies PF (1995) Flow-mediated endothelial mechanotransduction. Physiol Rev 75:519-560

38. Milkiewicz M, Uchida C, Gee E, Fudalewski T, Haas TL (2008) Shear stress-induced Ets-1 modulates protease inhibitor expression in microvascular endothelial cells. J Cell Physiol 217:502510. doi: $10.1002 / \mathrm{jcp} .21526$

39. Tressel SL, Huang RP, Tomsen N, Jo H (2007) Laminar shear inhibits tubule formation and migration of endothelial cells by an angiopoietin-2 dependent mechanism. Arterioscler Thromb Vasc Biol 27:2150-2156. doi:10.1161/ATVBAHA.107.150920

40. Franke RP, Grafe M, Schnittler H, Seiffge D, Mittermayer C, Drenckhahn D (1984) Induction of human vascular endothelial stress fibres by fluid shear stress. Nature 307:648-649. doi: $10.1038 / 307648 \mathrm{a} 0$

41. Davies PF, Remuzzi A, Gordon EJ, Dewey CF Jr, Gimbrone MA Jr (1986) Turbulent fluid shear stress induces vascular endothelial cell turnover in vitro. Proc Natl Acad Sci USA 83:2114-2117. doi:10.1073/pnas.83.7.2114

42. Ichioka S, Shibata M, Kosaki K, Sato Y, Harii K, Kamiya A (1998) In vivo measurement of morphometric and hemodynamic changes in the microcirculation during angiogenesis under chronic alpha1-adrenergic blocker treatment. Microvasc Res 55:165-174. doi:10.1006/mvre.1998.2069

43. Egginton S, Zhou AL, Brown MD, Hudlicka O (2001) Unorthodox angiogenesis in skeletal muscle. Cardiovasc Res 49:634646. doi:10.1016/S0008-6363(00)00282-0

44. Hudlicka O, Brown M, Egginton S (1992) Angiogenesis in skeletal and cardiac muscle. Physiol Rev 72:369-417

45. Rivilis I, Milkiewicz M, Boyd P, Goldstein J, Brown MD, Egginton $S$ et al (2002) Differential involvement of MMP-2 and VEGF during muscle stretch- versus shear stress-induced angiogenesis. Am J Physiol Heart Circ Physiol 283:H1430-H1438

46. Osawa M, Masuda M, Kusano K, Fujiwara K (2002) Evidence for a role of platelet endothelial cell adhesion molecule-1 in endothelial cell mechanosignal transduction: is it a mechanoresponsive molecule? J Cell Biol 158:773-785. doi:10.1083/jcb.200205049

47. Fisher AB, Chien S, Barakat AI, Nerem RM (2001) Endothelial cellular response to altered shear stress. Am J Physiol Lung Cell Mol Physiol 281:L529-L533

48. Zakrzewicz A, Secomb TW, Pries AR (2002) Angioadaptation: keeping the vascular system in shape. News Physiol Sci 17:197-201

49. Urbich C, Stein M, Reisinger K, Kaufmann R, Dimmeler S, Gille J (2003) Fluid shear stress-induced transcriptional activation of the vascular endothelial growth factor receptor-2 gene requires Sp1-dependent DNA binding. FEBS Lett 535:87-93. doi: 10.1016/S0014-5793(02)03879-6

50. Morbidelli L, Chang CH, Douglas JG, Granger HJ, Ledda F, Ziche M (1996) Nitric oxide mediates mitogenic effect of VEGF on coronary venular endothelium. Am J Physiol 270:H411-H415

51. Hudetz AG, Kiani MF (1992) The role of wall shear stress in microvascular network adaptation. Adv Exp Med Biol 316:31-39

52. Clarke ER, Clarke EL (1940) Microscopic observations on the extraendothelial cells of living mammalian blood vessels. Am J Anat 66:1-49. doi:10.1002/aja.1000660102

53. Zhang J, Cao R, Zhang Y, Jia T, Cao Y, Wahlberg E (2009) Differential roles of PDGFR- $\{$ alpha $\}$ and PDGFR- $\{$ beta $\}$ in angiogenesis and vessel stability. FASEB J 23:153-163

54. Benjamin LE, Hemo I, Keshet E (1998) A plasticity window for blood vessel remodelling is defined by pericyte coverage of the preformed endothelial network and is regulated by PDGF-B and VEGF. Development 125:1591-1598

55. Hellstrom M, Kalen M, Lindahl P, Abramsson A, Betsholtz C (1999) Role of PDGF-B and PDGFR-beta in recruitment of vascular smooth muscle cells and pericytes during embryonic blood vessel formation in the mouse. Development 126:3047-3055

56. Ohlsson R, Falck P, Hellstrom M, Lindahl P, Bostrom H, Franklin G et al (1999) PDGFB regulates the development of the labyrinthine layer of the mouse fetal placenta. Dev Biol 212:124136. doi:10.1006/dbio.1999.9306

57. Suri C, Jones PF, Patan S, Bartunkova S, Maisonpierre PC, Davis $S$ et al (1996) Requisite role of angiopoietin-1, a ligand for the TIE2 receptor, during embryonic angiogenesis. Cell 87:11711180. doi:10.1016/S0092-8674(00)81813-9

58. Uemura A, Ogawa M, Hirashima M, Fujiwara T, Koyama S, Takagi $\mathrm{H}$ et al (2002) Recombinant angiopoietin-1 restores higher-order architecture of growing blood vessels in mice in the absence of mural cells. J Clin Invest 110:1619-1628

59. Betsholtz C, Karlsson L, Lindahl P (2001) Developmental roles of platelet-derived growth factors. Bioessays 23:494-507. doi: 10.1002/bies. 1069

60. Thurston G, Suri C, Smith K, McClain J, Sato TN, Yancopoulos GD et al (1999) Leakage-resistant blood vessels in mice transgenically overexpressing angiopoietin-1. Science 286:25112514. doi:10.1126/science.286.5449.2511

61. Thurston G (2002) Complementary actions of VEGF and angiopoietins on blood vessel permeability and growth in mice. J Anat 200:529. doi:10.1046/j.1469-7580.2002.00061.x

62. Thurston G (2002) Complementary actions of VEGF and angiopoietin-1 on blood vessel growth and leakage. J Anat 200:575-580. doi:10.1046/j.1469-7580.2002.00061.x

63. Carmeliet P, Ferreira V, Breier G, Pollefeyt S, Kieckens L, Gertsenstein M et al (1996) Abnormal blood vessel development and lethality in embryos lacking a single VEGF allele. Nature 380:435-439. doi:10.1038/380435a0

64. Ferrara N, Carver-Moore K, Chen H, Dowd M, Lu L, O'Shea KS et al (1996) Heterozygous embryonic lethality induced by targeted inactivation of the VEGF gene. Nature 380:439-442. doi: $10.1038 / 380439 \mathrm{a} 0$ 